\title{
Hemodynamic changes in osteonecrosis treatment of the femoral head with iliac bone flaps pedicled with the lateral femoral circumflex artery ascending branch: A 10-year report
}

\author{
YuPeng Liu ${ }^{\mathrm{a}, \mathrm{b}}$, DeWei Zhao ${ }^{\mathrm{b}, *}$, W.M. Wang ${ }^{\mathrm{b}}$, B.J. Wang ${ }^{\mathrm{b}}$, Y. Zhang ${ }^{\mathrm{b}}$ and Z.G. Li ${ }^{\mathrm{b}}$ \\ ${ }^{a}$ Department of Biomedical Engineering, Dalian University of Technology, Dalian, Liaoning, China \\ ${ }^{\mathrm{b}}$ Affiliated Zhongshan Hospital of Dalian University, Dalian, Liaoning, China
}

\begin{abstract}
.
BACKGROUND: Vascularized bone grafting was used in the treatment of osteonecrosis femoral head, which may directly influence the pathologic event of femoral head.

OBJECTIVE: This paper evaluates hemodynamic changes in osteonecrosis treatment of the femoral head (ONFH) with iliac bone flaps from the lateral femoral circumflex artery ascending branches via digital subtraction angiography.

METHODS: A total of 48 patients, (31 males and 17 females; 38 at stage II and 10 at stage III), were treated with iliac bone flaps pedicled with the ascending branch of the lateral femoral circumflex artery. DSA was performed on all 48 patients preoperatively and 6 months postoperatively, and 10 years postoperatively on 1 patient.

RESULTS: Six months after surgery, femoral head blood supplies were distinctly visualized in the selective DSA in 44 cases. 4 patients showed poor or failed vessel pedicle filling in the transplanted bone flaps. Total hip arthroplasty was performed on these 4 patients. DSA was conducted 10 years post-operation in 1 case; the subject showed normal blood supply of the femoral head.

CONCLUSIONS: The anatomical position of the ascending branch of the lateral femoral circumflex artery was constant. Digital subtraction angiography successfully examined hemodynamic changes in osteonecrosis treatment of the femoral head with vascularized bone flaps.
\end{abstract}

Keywords: Femoral head necrosis, digital subtraction angiography, bone remodelling

\section{Introduction}

Osteonecrosis of the femoral head (ONFH) is a common disease; typical symptoms include a decrease in blood flow to the femoral head, and collapse of the articular surface. A variety of factors have been identified as risk factors for ONFH, but the etiology and pathogenesis still remains unclear [1-7].

\footnotetext{
${ }^{*}$ Corresponding author: Dewei Zhao, Department of Orthopaedic Surgery, Affiliated Zhongshan Hospital of Dalian University, No.6 Jiefang Street, Dalian 116001, Liaoning, China. Tel.: +86 041162893509; Mobile: +86 13390503299 ; E-mail: zhaodewei2000@163.com.
} 
Table 1

Properative patient demographics

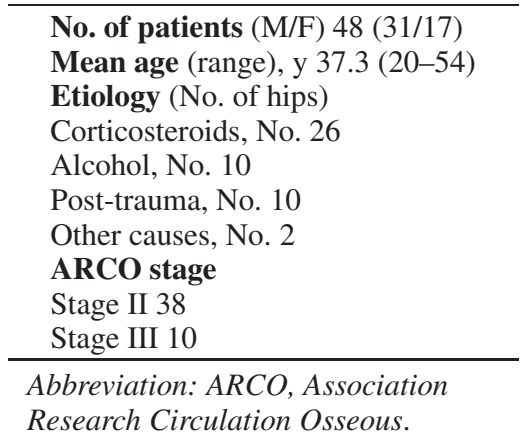

ONFH tends to affect younger patients; therefore, various head-preserving procedures have been used to delay the progression of the disease and provide pain relief to avert or delay total hip arthroplasty (THA) [8-13]. Femoral head sparing procedures (FHSP) include core decompression (CD), different bone grafting procedures, and/or biologic agents and rotational osteotomies. Non-vascularised bone grafting from different sources have been used to fill the necrotic area in the femoral head and provide structural support to the subchondral bone and articular cartilage, thereby preventing collapse during mechanism repair [14,15]. Vascularized bone grafting include the fibula [16] and iliac crest bone block transfer [17-20]. Currently, there is no consensus regarding the treatment of the different stages of ONFH to reliably prevent ONFH progression. Good results were obtained in $94 \%$ of patients younger than 45 years with Ficat and Arlet Stage II osteonecrosis of the femoral head [21]. However, digital subtraction angiography (DSA) was selected in this study to evaluate hemodynamic changes after femoral head osteonecrosis treatment $(\mathrm{ONFH})$ with the ascending branch of lateral femoral circumflex artery bone flaps.

\section{Materials and methods}

\subsection{Patients}

We retrospectively reviewed clinical data for 48 consecutive patients diagnosed with segmental femoral head necrosis who received treatment at the Affiliated ZhongShan Hospital of Dalian University between 1998 and 2008. The same surgeon operated on all patients (DWZ). These 48 patients included 31 males and 17 females between the ages of 20 and 54 (mean, 37.3 years). According to the ARCO classification, 38 were stage II and 10 were stage III. The etiological causes of osteonecrosis were steroid use in 26 patients, alcohol abuse in 10 patients, post-traumatic osteonecrosis in 10 patients, and other causes in 2 patients. Preoperative Harris hip scores ranged from 45 to 75 (mean, 56.0), as shown in Table 1.

\subsection{Materials}

Materials included a DSA system (Digital Fluorikon 5000, General Electric Co.), a 4 French tight J-curved Simmons 80-cm catheter, a 19-gauge extra-thin-wall Seldinger needle, and a J/Straight floppy 125-cm guidewire. 


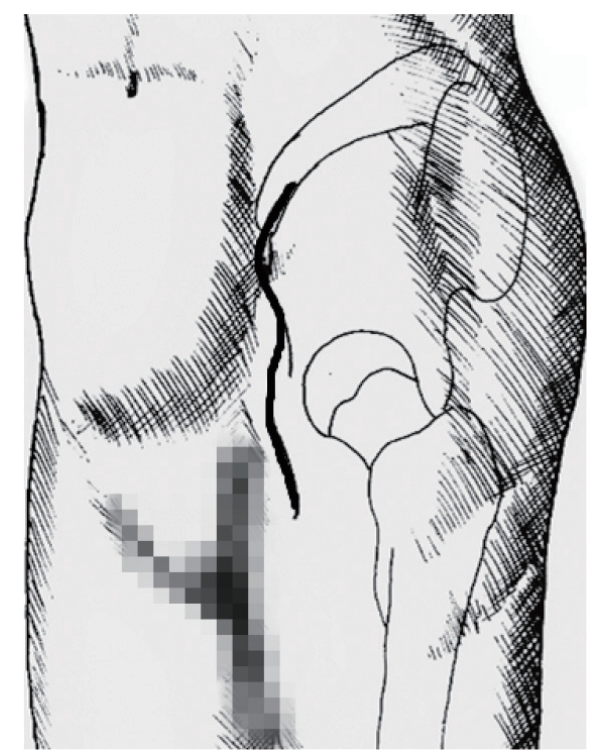

Fig. 1. The illustration shows the location of the $12-\mathrm{cm}$ double curve incision. It was $4 \mathrm{~cm}$ proximal to the anterosuperior iliac spine.

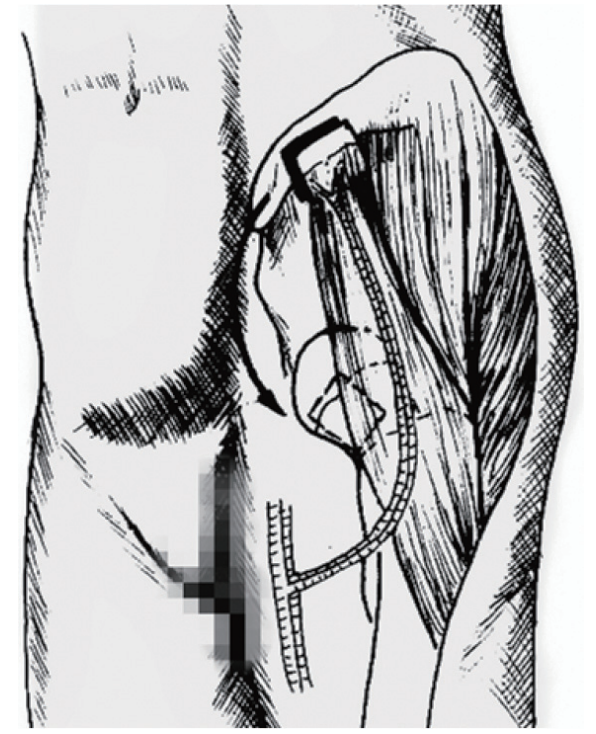

Fig. 2. The illustration shows a vascularised bone block, based on the ascending branch of the lateral femoral circumflex vessels.

\subsection{Methods}

\subsubsection{Operation method}

All patients were treated with iliac bone flaps pedicled with the ascending branch of the lateral femoral circumflex artery. Figures 1 and 2 show the process.

\subsubsection{DSA Method: Digital subtraction angiography of the femoral head using the Seldinger technique}

Paracentesis was performed from the normal-side femoral artery by inserting a 3-5F c-curved Simmons $80-\mathrm{cm}$ catheter into the external iliac artery of the affected side and selection of the lateral femoral circumflex artery and the medial femoral circumflex artery. Before this, $8 \mathrm{~mL}$ of iohexol at $4 \mathrm{~mL} / \mathrm{sec}$ and $300 \mathrm{kpa}$ pressure was injected; orthotope and double-sided oblique-position X-ray films were obtained. The vessel visualization, blood distribution, and staining of the affected femoral head were observed.

Selective DSA was performed on all 48 patients before operation and 6 months to 10 years postoperatively (mean 4.5 years). Selective DSA was performed in 1 of the 48 patients 10 years after the operation.

\section{Results}

DSA was performed on all 48 patients successfully, both preoperatively and postoperatively. Preoperative results demonstrated abnormal vascularisation in all 48 patients, extenuation breaks in the supraretinaculum arteries, and the loss of normal appearance, including diminished arteries. These diminished arteries were likely due to small artery revascularization. Hips (ARCO II, III) displayed abnormal femoral head venous return and showed color anomalies or time delays in different femoral head fields in the parenchyma period. The venous tributary decrease in the venous phase and slow movement of the 
contrast media was also accompanied by contrast media siltation. These observations demonstrate that the femoral head blood supply had predominantly been lost.

Selective DSA showed that the lateral femoral circumflex artery site was constant. The lateral femoral circumflex artery's ascending, transverse, and descending branches had sharp positions without large variations. The outside diameter of the ascending ramus origin part was $3.2 \pm 0.9 \mathrm{~mm}$, and its length was $8.5 \pm 3.1 \mathrm{~cm}$.

From six months after the operation, the ramus ascendens arteriae circumflexae femoris lateralis and the following veins were distinctly visualized in the selective DSA; the femoral head staining fields were enlarged while the staining time shortened. The number of blood vessels per unit area increased. Venous return visualization was faster after the operation than before, and it showed obvious improvements. These results demonstrate that the femoral head blood supply was reversing to a more normal state after the operation. Microcirculation was restored six month postoperatively. Selective DSA was performed in 1 patient 10 years after the operation; the results showed satisfactory filling. In 4 patients, poor filling and 2 failures of vessel pedicle filling in the transplanted bone flaps was observed 6 months postoperatively. 4 cases received a THA 1 and 2 years postoperation, including 2 failure filling cases and 2 poor filling cases. The clinical success rate of this group was $91.7 \%$. Selective DSA was performed in 1 of the 48 patients 10 years after the operation showed good filling.

\section{Discussion}

The blood flow of the femoral head mainly comes from the medial femoral circumflex artery. The upper-aboral retinacular artery supplies roughly $66 \%$ of the area of the femoral head. Femoral head necrosis can occur in multiple locations within this area. The anterior-posterior retinacular artery supplies $25-33 \%$ of the inferior femoral head field. The inferior retinacular artery entering the femoral head is small and has few branches. The round ligament artery only supplies the fossa capitis femoris field. The femur head and the supra-neck articular capsule artery, which provides $65-80 \%$ of the femoral head and sub-joint capsule artery blood supply, are visualized well and are sharply visible in normal DSA [22]. The inosculation of the medial femoral circumflex artery and obturator artery is also sharp. The lateral femoral circumflex artery passes through the linea intertrochanterica and supplies the outside soft tissues and vicinity muscles outside the femoral head. The superior gluteal artery and the inferior gluteal artery are visualized simultaneously in DSA.

Vascularized iliac bone graft, perfused by the lateral femoral circumflex artery ascending branch, has been used widely in China for approximately two decades as an alternative method for treating osteonecrosis, with satisfactory results [23]. Results of an anatomical study and clinical research [24,25] showed that the vascular branches that run into the anterosuperior iliac crest along the tensor fasciae lata muscles are consistently present. However, we suspect that a good blood supply postoperatively is critical, and that it helps to prevent femoral head blood supply occlusion.

Free vascularized fibular transplant and vascularized iliac crest transplants have been reported to improve blood supply functionality [26]. Some surgeons have considerable experience with free autogenous vasularized fibular transplantation, whereas others rely on transplants [27,28]. However, the necessity for microvascular anastomosis is a disadvantage to the free vascularized fibular transplant compared with the vascularized iliac crest transplant.

Starklint et al. [29] found in a study that vein specimens of the femoral head's bone marrow blood vascular system excised from advanced-stage ONFH were generally not suitable for immunohistochemical studies. Moreover, red blood cell accumulation and newly-arising or old fibrin thrombi were seen, and 
afferent fibers formed. Venous outflow tract blockage increased intraosseous pressure, slowed/ceased the venous system blood flow, and decreased the partial pressure of bone marrow oxygen. In our study, selective preoperative DSA showed mainly venous stasis in ARCO stage II, indicating that microcirculation disturbance and/or venous deposition and back flow obstruction occurred in the femoral head.

Abnormal arterial blood supply was the predominant representation of ARCO III stage of ONFH. The feeding artery was tiny or interrupted, indicating insufficient arterial blood supply. These signs were seen simultaneously in some ONFH patients. Selective DSA performed 6 months after the operation showed that in 42 of the 48 patients, the bone graft flap arteries were well-visualized, well-stacked, and had no interruptions. Furthermore, the femoral head staining field was enlarged, the staining time shortened, the number of blood vessels increased in unit area, the venous return visualization shifted to an earlier time, and the back flow obstruction was obviously improved. This data suggested that the femoral head blood supply was restored, and microcirculation restoration had begun. In this report, selective DSA performed before the operation provided direct evidence of the blood supply route. It is advisable to avoid exploring blood vessels or vascular injury during the operation due to anatomical variations to ensure desirable outcomes. In 6 of 48 cases, selective DSA performed before the operation showed the ramus ascendens arteriae circumflexae femoris lateralis filling well, but DSA performed after the operation showed that the transplanted bone flap with the ramus ascendens arteriae circumflexae femoris lateralis under-filled or had no filling with the contrast media in the femoral head. 4 of these 6 cases received THA due to vosospasm or bone graft flap vessel pedicle distortion during the operation, and due to other reasons that led to blood supply obstruction. It is not yet clear which specific development mechanism applied in each individual case; this area must be further studied. Our data suggests that femoral head blood supply reversion is key to successful ONFH treatment.

In summary, selective DSA can help visualize the precise femoral head blood supply and provide accurate information about the site and distribution of the lateral femoral circumflex artery, which is helpful for devising the operation strategy. It can also help reveal femoral head blood supply reversion in postoperative follow-up; this method is more objective than other evaluation methods. However, selective DSA has contraindications and pitfalls. DSA contraindications include:

(1) Iodine allergy

(2) Serious heart, liver, or kidney failure

(3) Blood coagulation dysfunction, or tendency to bleed

(4) Fever or period of acute inflammation

(5) Systemic infection or sepsis

(6) The patient is older than 55

(7) IV ARCO classification

Additionally, DSA is an expensive and traumatic procedure. Selective DSA should not be the standard method of ONFH diagnosis and follow-up.

\section{Acknowledgements}

My deepest gratitude goes first and foremost to Professor Dewei Zhao, my supervisor, for he constant encouragement and guidance. He has walked me through all the stages of the writing of this thesis.

Second, I would like to express my heartfelt gratitude to my team.

Last my thanks would go to my beloved family for their loving considerations and great confidence in me all through these years. 


\section{References}

[1] Jones LC, Hungerford DS. The pathogenesis of osteonecrosis. Instr Course Lect. 2007; 56: 179-96.

[2] Jones Jr JP. Concepts of etiology and early pathogenesis of osteonecrosis. Instr Course Lect. 1994; 43: 499-512.

[3] Matsuo K, Hirohata T, Sugioka Y, Ikeda M, Fukuda A. Influence of alcohol intake, cigarette smoking, and occupational status on idiopathic osteonecrosis of the femoral head. Clin Orthop Relat Res. 1988 Sep; (234): 115-23.

[4] Nishimura T, Matsumoto T, Nishino M, Tomita K. Histopathologic study of veins in steroid treated rabbits. Clin Orthop Relat Res. 1997 Jan; (334): 37-42.

[5] Nagi ON, Dhillon MS, et al. Open reduction, internal fixation and fibular autografting for neglected fracture of the femoral neck. J Bone Joint Surg (Br). 1998; 80-B: 794-804.

[6] Protzman RD, Burkhalter WE. Femoral-neck fractures in young adults. J Bone Joint Surg (Am). 1976; 58-A: 689-95.

[7] Moya-Angeler J, Gianakos AL, Villa JC, Ni A, Lane JM. Current concepts on osteonecrosis of the femoral head. World J Orthop. 2015; 6(8): 590-601.

[8] Mont MA, Einhorn TA, Sponseller PD, Hungerford DS. The trapdoor procedure using autogenous cortical and cancellous bone grafts for osteonecrosis of the femoral head. J Bone Joint Surg. 1998; 80B: 56-62.

[9] Mont MA, Etienne G, Ragland PS. Outcome of nonvascularized bone grafting for osteonecrosis of the femoral head. Clin Orthop Relat Res. 2003; 417: 84-92.

[10] Lieberman JR, Berry DJ, Mont MA, Aaron RK, Callaghan JJ, Rajadhyaksha AD, Urbaniak JR. Osteonecrosis of the hip: management in the 21st century. Instr Course Lect. 2003; 52: 337-355.

[11] Mont MA, Marulanda GA, Jones LC, Saleh KJ, Gordon N, Hungerford DS, Steinberg ME. Systematic analysis of classification systems for osteonecrosis of the femoral head. J Bone Joint Surg Am. 2006; 88 Suppl 3: 16-26.

[12] Aldridge JM, Urbaniak JR. Avascular necrosis of the femoral head: etiology, pathophysiology, classification, and current treatment guidelines. Am J Orthop (Belle Mead NJ). 2004; 33: 327-332.

[13] Lavernia CJ, Sierra RJ, Grieco FR. Osteonecrosis of the femoral head. J Am Acad Orthop Surg. 1999; 7: $250-261$.

[14] Judet H, Gilbert A. Long-term results of free vascularized fibular grafting for femoral head necrosis. Clin Orthop Relat Res. 2001; 386: 114-119.

[15] Rosenwasser MP, Garino JP, Kiernan HA, Michelsen CB. Long term follow-up of thorough debridement and cancellous bone grafting of the femoral head for avascular necrosis. Clin Orthop Relat Res. 1994; 306: 17-27.

[16] Urbaniak JR, Coogan PG, Gunneson EB, Nunley JA. Treatment of osteonecrosis of the femoral head with free vascularized fibular grafting: A long-term follow-up study of one hundred and three hips. J Bone Joint Surg. 1995; 77A: 681-694.

[17] Hungerford M, Shirley E, Khanuja P, Hungerford D. Osteotomies as treatment for osteonecrosis. Curr Opin Orthop. 2003; 14: 17-22.

[18] Rosenwasser MP, Garino JP, Kiernan HA, Michelsen CB. Long term follow-up of thorough debridement and cancellous bone grafting of the femoral head for avascular necrosis. Clin Orthop Relat Res. 1994; 306: 17-27.

[19] Berend KR, Gunneson EE, Urbaniak JR. Free vascularized fibular grafting for the treatment of postcollapse osteonecrosis of the femoral head. J Bone Joint Surg. 2003; 85A: 987-993.

[20] Buckley PD, Gearen PF, Petty RW. Structural bone grafting for early atraumatic avascular necrosis of the femoral head. J Bone Joint Surg. 1991; 73A: 1357-1364.

[21] Zhao D, Xu D, et al. Iliac Grate Vascularization for Femoral Head Osteonecrosis. Clin Orthoped Relat Res. 2006; 442: 171-179.

[22] Zhao Dewei, Yu xiaobing, Wang Benjie, et al. Digital subtraction angiography in selection of the vascularized greater trochanter bone grafting for treatment of osteonecrosis of femoral head. Microsurgery. 2013 Nov; 33(8): 656-659.

[23] Zhao D, et al. Vascularized periosteum, the bone and vascular bundle implantation to repair bone defects in rabbits. Chinese J Orthop. 1998; 18(5): 299-302.

[24] Harris WH. Traumatic arthritis of the hip after dislocation and acetabular fractures: treatment by mold arthroplasty: an end result study using a new method of result evaluation. J Bone Jo int Surg (Am). 1969; 51(4): 737-755.

[25] Zhao D, Wang W, et al. Applied anatomy for transposition of two periosteal flaps pedicled with iliac crest and anterior inferior iliac branches of ascending rarus of lateral femoral circumflex vesses. Chinese J Orthop. 1998e; 18(5): 298-302.

[26] Bozic KJ, Zurakowski D, Thornhill TS. Survivorship analysis of hips treated with core decompression for nontraumatic osteonecrosis of the femoral head. J Bone Joint Surg. 1999; 81A: 200-209.

[27] Hasegawa $\mathrm{Y}$, Iwata $\mathrm{H}$, Torii S, et al. Vascularized pedicle bonegrafting for nontraumatic avascular necrosis of the femoral head. Arch Orthop Trauma Surg. 1997; 116: 251-258.

[28] Zhao Dewei, Sui Guangzhi, Du Guojun, et al. Vascularized bone flap transfer of large rotor head of the treatment of different diseases [J]. Chinese J Orthop. 1995; 15(9): 591-593.

[29] Starklint H, Lausten GS, Arnoldi CC. Microvascular obstruction in avascular necrosis Immunohistochemistry of 14 femoral heads. Acta Orthop Scand. 1995 Feb; 66(1): 9-12. 